PROCEEDINGS OF THE

AMERICAN MATHEMATICAL SOCIETY

Volume 115, Number 3, July 1992

\title{
UTILITY FUNCTIONS ON PARTIALLY ORDERED TOPOLOGICAL GROUPS
}

\author{
JUAN CARLOS CANDEAL-HARO AND ESTEBAN INDURAIN-ERASO
}

(Communicated by Andrew M. Bruckner)

\begin{abstract}
Let $(X,+, \tau)$ be a locally compact abelian group endowed with a translation-invariant, strongly continuous, and separable strict partial ordering " $<$." Then, there exists a continuous numerical representation for " $<$." The proof leans on the concept of Haar measure.
\end{abstract}

\section{INTRODUCTION}

The numerical representation of preordered sets is a tool for decision making, where the preference of decision makers are formalized with the help of utility functions that transform preferences into numerical scales.

The problem of finding characterizations of the existence of a numerical representation on an ordered set was posed long ago by Cantor [1,2]. Classical works on this subject are those by Eilenberg [5], Nachbin [12], and Fishburn [6]. Further studies on the general case of preordered sets appear in Mehta [9, 10] and Herden [7, 8].

In the framework of Mathematical Economics, some techniques related to measure theory have been considered in the study of utility indicators by Neuefeind [13], Mount and Reiter [11], and Chichilnisky [3, 4].

\section{NUMERICAL REPRESENTATION OF A PARTIALLY ORDERED TOPOLOGICAL GROUP}

Let $(X,+, \tau)$ be a locally compact abelian group with Haar measure $\mu$, endowed with a translation-invariant strict partial ordering " $<$ " (i.e., " $<$ " is irreflexive and transitive). Let "0" be the null element for the group operation "+", and denote by $B_{0}$ a compact neighbourhood of 0 in $(X, \tau)$.

The order " $<$ " is said to be separable if there exists a countable subset $D \subset X$ such that for every $a<b, a, b \in X$, there exists $d \in D$ with $a<d<b$. It is called strongly continuous as regards $\tau$ (see Peleg [14]) if for every $x \in X$ the sets $L(x)=\{a \in X ; a<x\}$ and $G(x)=\{b \in X ; x<b\}$ are open in $(X, \tau)$. A numerical representation (or "utility function," in Economics) is a continuous

Received by the editors November 7, 1990.

1991 Mathematics Subject Classification. Primary 28C10, 54F05; Secondary 06A110, 90A10.

Key words and phrases. Topological group, translation-invariant partial ordering, Haar measure, utility function. 
map $u:(X, \tau) \rightarrow \mathbb{R}$ such that for every $a, b \in X, a<b \Rightarrow u(a)<u(b)$. Let $L$ be a subset of $X, \partial L$ its boundary, $\operatorname{Int}(L)$ its interior, $\mathrm{Cl}(L)$ its closure and $\mathscr{X}_{L}$ the characteristic function of $L$ in $X$.

Theorem. Let $(X,+, \tau)$ be a locally compact abelian group endowed with a translation-invariant, strongly continuous, and separable strict partial ordering " $<$." Then $(X,<, \tau)$ admits a continuous numerical representation.

Proof. Let us prove that there exists a map $A: X \rightarrow \mathbb{R}$ such that the function $U: X \rightarrow \mathbb{R}$, defined as $U(x)=\int_{x+B_{0}} A(\cdot) d \mu(\cdot)$, is a numerical representation of $(X,<, \tau)$. To construct $A$, first consider a subset $D=\left(d_{n}\right)_{n \in \mathbb{N}} \subset X$ corresponding to the separability of $<$. Consider a finite and strictly positive measure $\delta$ defined on $\mathscr{P}(\mathbb{N})$. Now, define the measure $\delta^{*}$ on $\mathscr{P}(D)$ by taking $\delta^{*}(S)=\delta(T)$, where $S=\left(d_{t}\right)_{t \in T}, S \subset D, T \subset \mathbb{N}$. Finally extend the measure $\delta^{*}$ to a measure $v$ on $\mathscr{P}(X)$ such that $v(E)=\delta^{*}(E \cap D), E \subset X$.

Define $A$ as $A(x)=\sup \{(v(L(d)) ; d \in D, d<x\}$. It is not hard to see that $A$ is lower-semicontinuous, hence $\mu$-measurable, and bounded. So the function $U(x)=\int_{x+B_{0}} A(\cdot) d \mu(\cdot)$ is well defined.

To see that $U$ represents $(X,<, \tau)$ take $x, y \in X$ such that $x<y$. Then, since " $<$ " is translation-invariant, it follows that $x+b<y+b$ for every $b \in B_{0}$, and by definition of the map $A$, we have $A(x+b)<A(y+b)$ $\left(b \in B_{0}\right)$. Therefore $U(x)<U(y)$. Now, observe that if $\left(x_{\alpha}\right)_{\alpha \in J}$ is a net on $X$ converging to $x_{0} \in X$, then $\mathscr{X}_{x_{\alpha}+B_{0}}$ converges to $\mathscr{X}_{x_{0}+B_{0}} \mu$-almost everywhere. To see this, take $z \in X, z \notin \partial\left(x_{0}+B_{0}\right)$. Notice that $\mu\left(\partial\left(x_{0}+B_{0}\right)\right)=0$. The following two cases may occur: (i) $z \notin \mathrm{Cl}\left(x_{0}+B_{0}\right)$; (ii) $z \in \operatorname{Int}\left(x_{0}+B_{0}\right)$.

Let us see that in both cases the net $\left(\mathscr{X}_{x_{\alpha}+B_{0}}(z)\right)_{\alpha \in J}$ converges to $\mathscr{X}_{x_{0}+B_{0}}(z)$. In case (i), assume that $\left[\mathscr{X}_{x_{\alpha}+B_{0}}(z)\right]_{\alpha \in J}$ does not converge to $\mathscr{X}_{x_{0}+B_{0}}(z)=0$. Then pick up a subnet $\left(x_{\beta}\right)_{\beta \in K} \subset\left(x_{\alpha}\right)_{\alpha \in J}$ and a family of points $\left(b_{\beta}\right)_{\beta \in K} \subset B_{0}$ such that $z=x_{\beta}+b_{\beta}(\beta \in K) \quad\left(\Rightarrow \mathscr{X}_{x_{\beta}+B_{0}}(z)=1\right)$. Since $B_{0}$ is compact and $\left(x_{\alpha}\right)_{\alpha \in J}$ is convergent, there is no loss of generality in assuming that the net $\left(b_{\beta}\right)_{\beta \in K}$ converges to some point $b_{0} \subset B_{0}$. Hence $z=x_{0}+b_{0} \in x_{0}+B_{0}$. Contradiction. In case (ii) observe that $z=x_{0}+b_{0}$ for some $b_{0} \in \operatorname{Int}\left(B_{0}\right)$. Thus, there exists $\alpha_{0} \in J$ such that for every $\alpha \geq \alpha_{0}$, it is $z=x_{\alpha}+b_{\alpha} \in x_{0}+B_{0}$ (being $\left.b_{\alpha}=b_{0}+\left(x_{0}-x_{\alpha}\right)\right)$. Therefore $\mathscr{X}_{x_{\alpha}+B_{0}}(z)=1=\mathscr{X}_{x_{0}+B_{0}}(z)$ for every $\alpha \geq \alpha_{0}$. Finally, let us check the continuity of $U$ : Consider a net $\left(x_{\alpha}\right)_{\alpha \in J}$ on $X$, convergent to $x_{0} \in X$. Take a compact subset $B \subset X$ and $\alpha_{0} \in J$ such that $x_{\alpha}+B_{0} \subset B$ for every $\alpha \geq \alpha_{0}$. Now apply the dominated convergence theorem and the previous fact on $\mu$-convergence to the functions $A(\cdot) \mathscr{X}_{x_{\alpha}+B_{0}}(\cdot)$ $\left(\alpha \geq \alpha_{0}\right)$ and conclude that the net $\left(U\left(x_{\alpha}\right)\right)_{\alpha \in J}$ converges to $U\left(x_{0}\right)$.

Remark. The above result can be extended to the case of complete preorderings for which the indifference classes are $\mu$-negligible.

\section{REFERENCES}

1. G. Cantor, Beiträge zur Begründung der transfinite Mengenlehre I, Math. Ann. 46 (1895), 481-512.

2. $\frac{}{246}$, Beiträge zur Begründung der transfinite Mengenlehre II, Math. Ann. 49 (1897), 207-

3. __ Spaces of economic agents, J. Econom. Theory 15 (1977), 160-173. 
4. G. Chichilnisky, Continuous representations of preferences, Rev. Econom. Stud. 47 (1980), 959-963.

5. S. Eilenberg, Ordered topological spaces, Amer. J. Math. 63 (1941), 39-45.

6. P. C. Fishburn, Utility theory for decision making, John Wiley, New York, 1970.

7. G. Herden, On the existence of utility functions I, Math. Social Sci. 17 (1989), 297-313.

8. _ On the existence of utility functions II, Math. Social Sci. 18 (1989), 107-117.

9. G. Mehta, Continuous utility functions, Econom. Lett. 18 (1985), 113-115.

10. _ Existence of an order-preserving function on normally preordered spaces, Bull. Austral. Math. Soc. 34 (1986), 141-147.

11. K. R. Mount and S. Reiter, Construction of a continuous utility function for a class of preferences, J. Math. Econom. 3 (1976), 227-245.

12. L. Nachbin, Topologia e ordem, Univ. of Chicago Press, 1950.

13. W. Neuefeind, On continuous utility, J. Econom. Theory 5 (1972), 174-176.

14. B. Peleg, Utility functions for partially ordered topological spaces, Econometrica 38 (1970), 93-96.

Departamento de Mathematicas, Universidad de Zaragoza, Spain

Current address: Departamento de Análisis Económico, Facultad de Ciencias Económicas y Empresariales, C/Gran Via 2-4, 50005-Zaragoza, Spain

Departamento de Mathematicas, Universidad Pública de Navarra, Pamplona, Spain

Current address: Departamento de Matemáticas e Informática, C/ El Sadar s/n, 31006 Pamplona Spain 